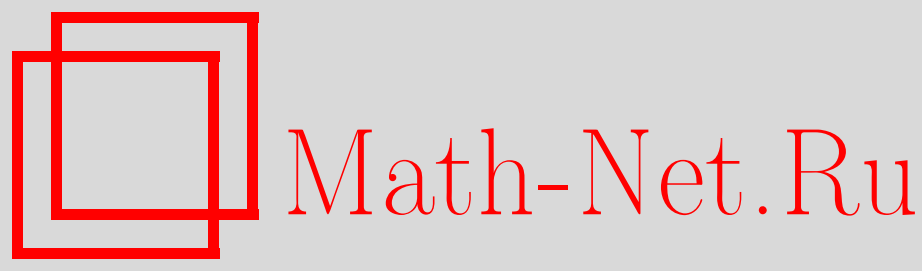

Л. Д. Фаддеев, Новые динамические переменные теории тяготения Эйнштейна, ТMФ, 2011, том 166, номер 3, 323-335

DOI: https://doi.org/10.4213/tmf6614

Использование Общероссийского математического портала Math-Net.Ru подразумевает, что вы прочитали и согласны с пользовательским соглашением http://www . mathnet.ru/rus/agreement

Параметры загрузки:

IP : 3.82 .47 .9

26 апреля 2023 г., $16: 25: 29$

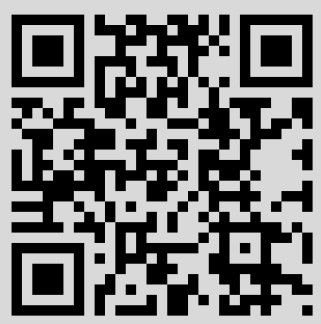




\section{НОВЫЕ ДИНАМИЧЕСКИЕ ПЕРЕМЕННЫЕ ТЕОРИИ ТЯГОТЕНИЯ ЭЙНШТЕЙНА}

Описан альтернативный формализм теории тяготения Эйнштейна. Роль динамических переменных играет набор из десяти векторных полей $f_{\mu}^{A}, A=$ $1, \ldots, 10$. Метрика является композитной переменной, $g_{\mu \nu}=f_{\mu}^{A} f_{\nu}^{A}$. Предложенная схема может дать дальнейшее развитие теории тяготения, в котором теория Эйнштейна будет играть роль эффективной теории, и константа Ньютона появится посредством введения аномальной функции Грина.

Ключевые слова: теория тяготения Эйнштейна, векторные поля, гамильтонова формулировка.

\section{1. ВВЕДЕНИЕ}

Теория тяготения Эйнштейна [1] является величайшим достижением теоретической физики XX века. Ее создание из априорных принципов, опередившее экспериментальное подтверждение, представляет собой триумф теоретического подхода к изучению природы. Геометрические конструкции, лежащие в основе ее формулировки, замечательно иллюстрируют роль математики в описании фундаментальных законов физики.

Теория тяготения появилась как классическая теория поля; во время ее появления другим примером была только электродинамика. Создание квантовой механики и достаточно успешная формулировка квантовой электродинамики, а также появление других квантовых теоретико-полевых построений поставили задачу квантования теории тяготения. Начиная с фундаментальной работы Дирака [2] проблеме квантования гравитации было посвящено множество подходов и предложений. Мы сейчас знаем гамильтонову интерпретацию теории Эйнштейна и ее формальное квантование в рамках формализма континуального интеграла (см. работу [3] и приведенную в ней литературу). Однако результат все еще неудовлетворителен, и проблема квантования не решена. Техническая трудность состоит в том, что соответствующая теория возмущений неперенормируема. В процессе перенормировки появляются контрчлены, не входящие в классический лагранжиан. Формальной

* Санкт-Петербургское отделение Математического института им. В. А. Стеклова РАН, Санкт-Петербург, Россия. E-mail: faddeev@pdmi.ras.ru 
причиной этого является тот факт, что константа связи (постоянная Ньютона) имеет размерность длины, $[\kappa]=[\mathrm{L}]$, и высшие степени разложения по $\kappa$ могут иметь коэффициенты с высшими производными от фундаментальных полей. Это обстоятельство отличает теорию тяготения Эйнштейна от электродинамики и теории Янга-Миллса, в которых константы связи безразмерны, расходимости логарифмические и количество безразмерных контрчленов конечно.

Схожная трудность, существовавшая в четырехфермионной теории слабых взаимодействий, была решена в теории Вайнберга-Салама [4], [5], где вместо размерной константы связи фигурирует поле Хиггса. Естественно, что аналогичные попытки предпринимались и в теории тяготения (см. обзор [6]), однако вполне удовлетворительной схемы все еще нет.

Настоящая работа представляет собой еще одну попытку найти новую схему, в которую теория Эйнштейна входила бы как эффективная теория. Имея в виду успех теории Вайнберга-Салама, я решил искать формулировку теории тяготения в терминах векторных полей. Пример такой формулировки уже существует. Он основан на теории погружения, согласно которой метрика четырехмерного пространства интерпретируется как индуцированная метрика при вложении этого пространства в десятимерное пространство:

$$
g_{\mu \nu}=f_{\mu}^{A} f_{\nu}^{A}, \quad f_{\mu}=\partial_{\mu} f^{A},
$$

где $f^{A}, A=1, \ldots, 10,-$ координаты погружения. Работы [7], [8] дают достаточно полную информацию об этом подходе.

Однако уравнения движения в этой теории отличаются от уравнений Гильберта-Эйнштейна. Действительно,

$$
\delta S=\delta\left(\int \sqrt{g} R d^{4} x\right)=\int \delta g_{\mu \nu} G^{\mu \nu} d^{4} x=-2 \int \partial_{\nu}\left(G^{\mu \nu} \partial_{\mu} f^{A}\right) \delta f^{A} d^{4} x
$$

так что соответствующее уравнение движения содержит производные тензора Риччи $G^{\mu \nu}$. Это обстоятельство послужило поводом к разочарованию в этом подходе для авторов работы [7], в то время как авторы работы [8] приводят основательные аргументы в его поддержку.

Мне очень понравились главные формулы теории погружения, и я решил их модифицировать, считая $f_{\mu}^{A}$ независимыми векторными полями, а не градиентами скаляров. Это должно было помочь избежать лишних производных. Получившийся формализм описан в заметках [9], [10]. Окончательный результат состоит в том, что возникшая схема эквивалентна теории Эйнштейна. Поэтому возникает естественный вопрос, зачем делать такую переформулировку? Мой ответ состоит в том, что новая схема может стать более естественным отправным пунктом для поиска более фундаментальной теории.

В разделе 2 настоящей статьи приводятся необходимые сведения из дифференциальной геометрии. В разделе 3 выписаны уравнения движения и обсуждается их эквивалентность уравнениям Гильберта-Эйнштейна. Раздел 4 содержит гамильтонову редукцию основного лагранжиана. В разделе 5 обсуждаются возможные направления обобщения теории тяготения Эйнштейна. 


\section{2. НЕКОТОРЫЕ ФОРМУЛЫ ДИФФЕРЕНЦИАЛЬНОЙ ГЕОМЕТРИИ}

На протяжении всей работы я буду использовать евклидову сигнатуру, отличая пространство и время только в обозначениях для координат $x^{\mu}=\left(x^{0}, x^{k}\right)$. Это позволит мне не загружать формулы лишними знаками минус. Переход к псевдоевклидовой структуре происходит почти автоматически.

Пусть $M^{4}$ - четырехмерное пространство с локальными координатами $x^{\mu}, \mu=$ $0,1,2,3$. Динамические переменные составляют набор ковариантных векторных полей $f_{\mu}^{A}(x), A=1, \ldots, D$, где $D$ - достаточно большое число. В дальнейшем окажется, что следует взять $D=10$.

Инфинитезимальные преобразования координат $\delta x^{\mu}=\epsilon^{\mu}(x)$ действуют на $f_{\mu}^{A}$ обычным образом:

$$
\delta f_{\mu}^{A}=-\partial_{\mu} \epsilon^{\lambda} f_{\lambda}^{A}-\epsilon^{\lambda} \partial_{\lambda} f_{\mu}^{A} .
$$

Матрица Грама $g_{\mu \nu}=f_{\mu}^{A} f_{\nu}^{A}$ задает метрику на $M^{4}$, а такой объект, как

$$
\Omega_{\beta \mu}^{\alpha}=f^{\alpha A} \partial_{\mu} f_{\beta}^{A}=g^{\alpha \sigma} \Omega_{\sigma, \beta \mu}=g^{\alpha \sigma} f_{\sigma}^{A} \partial_{\mu} f_{\beta}^{A},
$$

где, как всегда, $f^{\alpha A}=g^{\alpha \beta} f_{\beta}^{A}, g^{\alpha \mu} g_{\mu \beta}=\delta_{\beta}^{\alpha}$, определяет линейную связность. Действительно, при координатных преобразованиях матрица $g_{\mu \nu}$ преобразуется как ковариантный тензор, а в вариацию $\Omega_{\beta \mu}^{\alpha}$, помимо обычных тензорных слагаемых, входит вторая производная от $\epsilon^{\alpha}$ :

$$
\delta \Omega_{\beta \mu}^{\alpha}=\cdots+\partial_{\mu} \partial_{\beta} \epsilon^{\alpha}
$$

Связность $\Omega_{\beta \mu}^{\alpha}$ совместима с метрикой $g_{\mu \nu}$, поскольку

$$
\begin{aligned}
\nabla_{\lambda} g_{\mu \nu} & =\partial_{\lambda} f_{\mu}^{A} f_{\nu}^{A}+f_{\mu}^{A} \partial_{\lambda} f_{\nu}^{A}-\Omega_{\mu \lambda}^{\sigma} g_{\sigma \nu}-\Omega_{\nu \lambda}^{\sigma} g_{\mu \sigma}= \\
& =\partial_{\lambda} f_{\mu}^{A} f_{\nu}^{A}+f_{\mu}^{A} \partial_{\lambda} f_{\nu}^{A}-\partial_{\lambda} f_{\mu}^{A} f^{\sigma A} g_{\sigma \nu}-\partial_{\lambda} f_{\nu}^{A} f^{\sigma A} g_{\mu \sigma}=0
\end{aligned}
$$

однако она не является римановой, и кручение

$$
T_{\alpha[\mu, \nu]}=\Omega_{\alpha, \mu \nu}-\Omega_{\alpha, \nu \mu}
$$

отлично от нуля. Риманова связность $\Gamma_{\alpha, \beta \mu}$ выражается через $\Omega_{\alpha, \beta \mu}$ следующим образом:

$$
\Gamma_{\alpha, \beta \mu}=\frac{1}{2}\left(\Omega_{\alpha, \beta \mu}+\Omega_{\alpha, \mu \beta}\right)+\frac{1}{2}\left(\Omega_{\mu, \alpha \beta}-\Omega_{\mu, \beta \alpha}\right)+\frac{1}{2}\left(\Omega_{\beta, \alpha \mu}-\Omega_{\beta, \mu \alpha}\right),
$$

так что $\Gamma$ и $\Omega$ совпадают только при условии, что кручение $T$ исчезает.

Вычислим тензор кривизны $S_{\beta, \mu \nu}^{\alpha}$ связности $\Omega_{\beta \mu}^{\alpha}$. Имеем по определению

$$
\begin{aligned}
S_{\beta, \mu \nu}^{\alpha} & =\partial_{\mu} \Omega_{\beta \nu}^{\alpha}-\partial_{\nu} \Omega_{\beta \mu}^{\alpha}+\Omega_{\sigma \mu}^{\alpha} \Omega_{\beta \nu}^{\sigma}-\Omega_{\sigma \nu}^{\alpha} \Omega_{\beta \mu}^{\sigma}= \\
& =\partial_{\mu}\left(f^{\alpha A} \partial_{\nu} f_{\beta}^{A}\right)-\partial_{\nu}\left(f^{\alpha A} \partial_{\mu} f_{\beta}^{A}\right)+f^{\alpha A} \partial_{\mu} f_{\sigma}^{A} f^{\sigma B} \partial_{\nu} f_{\beta}^{B}-f^{\alpha A} \partial_{\nu} f_{\sigma}^{A} f^{\sigma B} \partial_{\mu} f_{\beta}^{B}
\end{aligned}
$$

Вторые производные сокращаются; используя элементарное равенство

$$
f^{\alpha A} \partial_{\mu} f_{\sigma}^{A}=-\partial_{\mu} f^{\alpha A} f_{\sigma}^{A}
$$


которое следует из условия ортонормированности $f_{\mu}^{A} f^{\nu A}=\delta_{\mu}^{\nu}$, мы можем переписать $S_{\beta, \mu \nu}^{\alpha}$ следующим образом:

$$
S_{\beta, \mu \nu}^{\alpha}=\Pi^{A B}\left(\partial_{\mu} f^{\alpha A} \partial_{\nu} f_{\beta}^{B}-\partial_{\nu} f^{\alpha A} \partial_{\mu} f_{\beta}^{B}\right),
$$

где $\Pi^{A B}=\delta^{A B}-f_{\sigma}^{A} f^{\sigma B}$ есть проектор на $(D-4)$-мерное подпространство в $\mathbb{R}^{D}$, ортогональное $f_{\mu}^{A}$, рассматриваемым как векторы в $\mathbb{R}^{D}$ :

$$
\Pi^{A B} f_{\mu}^{A}=0=\Pi^{A B} f^{\mu A} .
$$

В дальнейшем я буду называть $\Pi^{A B}$ вертикальным проектором.

Хотя выражение $S_{\beta, \mu \nu}^{\alpha}$ содержит только локальные производные, оно общековариантно. Действительно, нековариантные слагаемые в $\partial_{\mu} f_{\alpha}^{A}$ пропорциональны $f_{\alpha}^{A}$ и обращаются в ноль при действии на них вертикального проектора. Это же соображение позволяет элементарно опустить верхний индекс в $S_{\beta, \mu \nu}^{\alpha}$, так что

$$
S_{\alpha \beta, \mu \nu}=g_{\alpha \sigma} S_{\beta, \mu \nu}^{\sigma}=\Pi^{A B}\left(\partial_{\mu} f_{\alpha}^{A} \partial_{\nu} f_{\beta}^{B}-\partial_{\nu} f_{\alpha}^{A} \partial_{\mu} f_{\beta}^{B}\right) .
$$

Теперь мы можем дать явно ковариантную запись для тензора кривизны. Введем тензор

$$
b_{\alpha \mu}^{A}=\nabla_{\mu} f_{\alpha}^{A}=\partial_{\mu} f_{\alpha}^{A}-\Omega_{\alpha \mu}^{\beta} f_{\beta}^{A}=\partial_{\mu} f_{\alpha}^{A}-f^{\beta B} \partial_{\mu} f_{\alpha}^{B} f_{\beta}^{A}=\Pi^{A B} \partial_{\mu} f_{\alpha}^{B} .
$$

Используя свойство проектора $\Pi^{A B}=\Pi^{A C} \Pi^{C B}$, мы можем записать $S_{\alpha \beta, \mu \nu}$ следующим образом:

$$
S_{\alpha \beta, \mu \nu}=b_{\alpha \mu}^{A} b_{\beta \nu}^{A}-b_{\alpha \nu}^{A} b_{\beta \mu}^{A},
$$

откуда очевидно вытекает свойство антисимметрии при перестановках $\alpha \leftrightarrow \beta$ и $\mu \leftrightarrow \nu$. Однако симметрии по отношению к перестановке $(\alpha \beta) \leftrightarrow(\mu \nu)$ нет. Заметим, впрочем, что для вычисления вариации использование локальных производных более удобно.

Объекты $\Omega_{\beta \mu}^{\alpha}$ и $b_{\beta \mu}^{A}$ являются горизонтальной и вертикальной проекциями производной $\partial_{\mu} f_{\beta}^{A}$,

$$
\partial_{\mu} f_{\beta}^{A}=\Omega_{\beta \mu}^{\sigma} f_{\sigma}^{A}+b_{\beta \mu}^{A} .
$$

Функция Лагранжа задается через скалярную кривизну $S$,

$$
\mathcal{L}=\sqrt{g} S=\sqrt{g} g^{\mu \alpha} g^{\nu \beta} S_{\alpha \beta, \mu \nu}=\sqrt{g} \Pi^{A B}\left(\partial_{\mu} f^{\mu A} \partial_{\nu} f^{\nu B}-\partial_{\nu} f^{\mu A} \partial_{\mu} f^{\nu B}\right),
$$

и представляет собой квадратичную форму от первых производных контравариантных компонент полей $f_{\mu}^{A}$.

Приведенные формулы схожи с формулой Гаусса, выражающей тензор кривизны подмногообразия через вторую квадратичную форму, однако тензор второй формы $b_{\alpha \mu}^{A}$ в нашем случае не симметричен.

\section{3. УРАВНЕНИЯ ДВИЖЕНИЯ}

В лагранжиане $\mathcal{L}$, приведенном выше, в качестве динамических переменных взяты контравариантные поля $f^{\mu A}$. Запишем вариацию действия в следующем виде:

$$
\delta \int \mathcal{L} d^{4} x=\int \Sigma_{\alpha}^{A} \delta f^{\alpha A} d^{4} x .
$$


Здесь $\Sigma_{\alpha}^{A}$ дает нам набор ковариантных векторных полей. С помощью прямых вычислений получаем следующее выражение для этих полей:

$$
\Sigma_{\alpha}^{C}=\sqrt{g}\left(2 Q_{\mu}^{A B C} S_{\alpha}^{\mu A B}-Q_{\alpha}^{A B C} S^{A B}\right),
$$

где

$$
\begin{aligned}
Q_{\mu}^{A B C} & =\Pi^{A B} f_{\mu}^{C}+\Pi^{A C} f_{\mu}^{B}+\Pi^{B C} f_{\mu}^{A}, \\
S_{\alpha}^{\mu A B} & =\partial_{\alpha} f^{\mu A} \partial_{\nu} f^{\nu B}-\partial_{\nu} f^{\mu A} \partial_{\alpha} f^{\nu B}, \\
S^{A B} & =\partial_{\mu} f^{\mu A} \partial_{\nu} f^{\nu B}-\partial_{\nu} f^{\mu A} \partial_{\mu} f^{\nu B} .
\end{aligned}
$$

Объект $S_{\alpha}^{\mu A B}$ не ковариантен, но его вклад в $\Sigma_{\alpha}^{A}$ ковариантен благодаря коэффициенту $Q_{\mu}^{A B C}$.

Рассмотрим в отдельности вертикальную и горизонтальную части $\Sigma_{\alpha}^{A}$ :

$$
\Sigma_{\alpha}^{A}=2 \sqrt{g}\left(f^{\mu A} H_{\alpha \mu}+\Pi^{A B} V_{\alpha}^{B}\right) .
$$

Вертикальная часть $V_{\alpha}^{A}$ имеет такой явный вид:

$$
V_{\alpha}^{A}=\sqrt{g}\left(\left(\Pi^{A C} f_{\mu}^{B}+\Pi^{A B} f_{\mu}^{C}\right) S_{\alpha}^{\mu B C}-\Pi^{A C} f_{\alpha}^{B} S^{B C}\right) .
$$

При выводе этого соотношения мы использовали симметрию тензора $Q_{\mu}^{A B C}$. С учетом определений $\Omega_{\beta \mu}^{\alpha}$ и $b_{\beta \mu}^{A}$ мы можем переписать $V_{\alpha}^{A}$ следующим образом:

$$
V_{\alpha}^{A}=b_{\beta}^{\beta A}\left(\Omega_{\mu \alpha}^{\mu}-\Omega_{\alpha \mu}^{\mu}\right)+b_{\alpha}^{\beta A}\left(\Omega_{\beta \mu}^{\mu}-\Omega_{\mu \beta}^{\mu}\right)+b_{\sigma}^{\beta A}\left(\Omega_{\alpha \beta}^{\sigma}-\Omega_{\beta \alpha}^{\sigma}\right) .
$$

Мы видим, что это выражение представляет собой линейную комбинацию компонент тензора кручения связности $\Omega_{\beta \mu}^{\alpha}$.

Индекс $A$ в системе уравнений $V_{\alpha}^{A}=0$ эффективно пробегает $D-4$ значений, так что всего мы имеем $4(D-4)$ вертикальных уравнений движения. С другой стороны, мы имеем $4 \cdot 6=24$ компонент кручения. При $D=10$ эти два числа совпадают, и вертикальная система уравнений становится линейной системой для $T_{[\mu, \nu]}^{\alpha}$ с квадратной $(24 \times 24)$-матрицей, составленной из коэффициентов $b_{\beta \mu}^{A}$. Записать определитель этой матрицы в аналитическом виде все еще является задачей, недоступной для компьютеров. Однако численный эксперимент, проведенный С. Пастоном, показал, что при выборе случайных значений для коэффициентов $b_{\beta \mu}^{A}$ этот определитель не равен нулю. В результате я предлагаю положить $D=10$ и считать, что вертикальные уравнения движения эквивалентны исчезновению кручения: $T_{[\mu, \nu]}^{\alpha}=0$.

Теперь перейдем к горизонтальной части уравнений движения

$$
H_{\alpha \mu}=\sqrt{g} \Pi^{B C}\left(S_{\alpha \mu}^{B C}-\frac{1}{2} g_{\alpha \mu} S^{B C}\right),
$$

которая означает исчезновение тензора Риччи,

$$
S_{\alpha \mu}-\frac{1}{2} g_{\alpha \mu} S=0
$$

где $S_{\alpha \mu}=g^{\nu \beta} S_{\alpha \beta, \mu \nu}$. Как мы видели выше, связности $\Omega_{\beta \mu}^{\alpha}$ и $\Gamma_{\beta \mu}^{\alpha}$ при исчезновении кручения совпадают, таким образом, тензор $S_{\alpha \mu}$ становится симметричным, 6 уравнений из 16 горизонтальных уравнений выполняются тривиально, а остальные 10 совпадают с уравнениями Гильберта-Эйнштейна. 
Мы видим, что действительно наш подход дает еще одну формулировку теории тяготения Эйнштейна.

В заключение этого раздела подчеркнем, что в наши уравнения движения входят только первые производные векторных полей $f_{\alpha}^{A}$. На первый взгляд, это противоречит утверждаемой нами эквивалентности. Действительно, уравнения Гильберта-Эйнштейна представляют собой систему уравнений второго порядка для метрики $g_{\mu \nu}$. Выход из этого парадокса дает следующее утверждение: риманов тензор кривизны $R_{\alpha \beta, \mu \nu}$ содержит вторые производные только через компоненты кручения $T_{[\mu, \nu]}^{\alpha}$. Действительно, вторые производные от $g_{\mu \nu}$ входят в $R_{\alpha \beta, \mu \nu}$ линейно в комбинации

$$
A_{\alpha \beta, \mu \nu}=\partial_{\mu} \partial_{\beta} g_{\nu \alpha}+\partial_{\nu} \partial_{\alpha} g_{\mu \beta}-\partial_{\mu} \partial_{\alpha} g_{\nu \beta}-\partial_{\nu} \partial_{\beta} g_{\mu \alpha}
$$

Рассмотрим выражение

$$
B_{\alpha \beta, \mu \nu}=\partial_{\mu}\left(T_{\alpha[\beta, \nu]}+T_{\nu[\beta, \alpha]}-T_{\beta[\alpha \nu]}\right)-\partial_{\nu}\left(T_{\alpha[\beta, \mu]}+T_{\mu[\beta, \alpha]}-T_{\beta[\alpha \mu]}\right) .
$$

Прямой проверкой можно убедиться, что сумма $A_{\alpha \beta, \mu \nu}+B_{\alpha \beta, \mu \nu}$ не содержит вторых производных от $f_{\mu}^{A}$, что и доказывает приведенное выше утверждение.

\section{4. (3 + 1)-РАЗДЕЛЕНИЕ ПЕРЕМЕННЫХ}

В этом разделе мы перепишем основные формулы, используя явное выделение одной из четырех координат $x^{\mu}=\left(x^{0}, x^{k}\right), k=1,2,3$. В случае лоренцевой сигнатуры это означает отделение времени $x^{0}$ от пространственных координат $x^{k}$. В евклидовой формулировке, которой мы следуем в этой работе, такое выделение одной координаты имеет более условный характер, однако, как я уже упомянул выше, переход к лоренцевой сигнатуре является почти автоматическим.

Итак, мы имеем набор динамических переменных $f_{0}^{A}, f_{k}^{A}, k=1,2,3$. По отношению к трехмерным координатным преобразованиям, полученным из четырехмерных $\epsilon^{\mu}(x)$ при ограничениях $\epsilon^{0}(x)=0, \partial_{0} \epsilon^{i}(x)=0$, эти переменные дают набор ковариантных векторов $\delta f_{k}^{A}=-\partial_{k} \epsilon^{l} f_{l}^{A}-\epsilon^{l} \partial_{l} f_{k}^{A}$ и скаляров $\delta f_{0}^{A}=-\epsilon^{l} \partial_{l} f_{0}^{A}$. Введем трехмерную метрику $\gamma_{i k}=f_{i}^{A} f_{k}^{A}$; пусть $\gamma^{i k}-$ соответствующий контравариантный тензор, $\gamma^{i k} \gamma_{l n}=\delta_{n}^{i}$. Компоненты $g^{i k}$ четырехмерной метрики и ее определитель выглядят так:

$$
g^{i k}=\gamma^{i k}+\frac{g^{0 i} g^{0 k}}{g^{00}}, \quad g=\frac{\gamma}{g^{00}},
$$

где $\gamma=\operatorname{det} \gamma_{i k}$.

Далее мы увидим, что вместо $f_{0}^{A}$ удобнее использовать соответствующее контравариантное поле $f^{0 A}=g^{00} f_{0}^{A}+g^{0 k} f_{k}^{A}$ и считать динамическими переменными набор $f_{k}^{A}, g^{0 k}, g^{00}$ и $f^{0 A}$. Полное число компонент в этом наборе равно 44 , однако они удовлетворяют четырем связям $g^{00}=f^{0 A} f^{0 A}, f^{0 A} f_{k}^{A}=0$, так что число независимых переменных равно 40.

Запишем функцию Лагранжа в виде

$$
\sqrt{g} S=\Sigma+\mathcal{H}
$$

где в $\Sigma$ собраны члены с производной по $x^{0}$, а $\mathcal{H}$ этих производных не содержит. Замечательно, что производные по $x^{0}$ входят в $\Sigma$ линейно,

$$
\Sigma=2 \sqrt{g} \Pi^{A B} g^{\mu l}\left(\partial_{l} f_{\mu}^{A} \partial_{0} f^{0 B}-\partial_{l} f^{0 A} \partial_{0} f_{\mu}^{B}\right),
$$


и $\mathcal{H}$ представляет собой квадратичную форму от пространственных производных,

$$
\mathcal{H}=\sqrt{g} g^{k \sigma} g^{l \rho}\left(\partial_{k} f_{\sigma}^{A} \partial_{l} f_{\rho}^{B}-\partial_{l} f_{\sigma}^{A} \partial_{k} f_{\rho}^{B}\right) .
$$

Мы можем интерпретировать $\Sigma$ как 1-форму при подстановке $\partial_{0} f_{\mu}^{A} \rightarrow d f_{\mu}^{A}$. Тогда функция Лагранжа приобретает канонический вид: 1-форма + 0-форма $d t$. Общий подход к приведению таких лагранжианов к обобщенному гамильтонову виду описан в работе [11] (см. также [12]). Мы применим этот подход к нашему примеру. Как показано в [11], при таком приведении можно использовать уравнения движения, не содержащие производных по времени. В нашем случае такими уравнениями являются соотношения

$$
\Omega_{m n}^{0}=\Omega_{n m}^{0}, \quad \Omega_{m n}^{i}=\Omega_{n m}^{i}
$$

Итак, приступим к приведению, начиная с 1-формы $\Sigma$. Запишем ее более подробно:

$$
\begin{aligned}
\Sigma=2 & \sqrt{g} \Pi^{A B}\left[\left(\gamma^{m l}+\frac{g^{0 m} g^{0 l}}{g^{00}}\right)\left(\partial_{l} f_{m}^{A} \partial_{0} f^{0 B}-\partial_{l} f^{0 A} \partial_{0} f_{m}^{B}\right)+\right. \\
& \left.+g^{0 l}\left(\partial_{l} f_{0}^{A} \partial_{0} f^{0 B}-\partial_{l} f^{0 A} \partial_{0} f_{0}^{B}\right)\right] .
\end{aligned}
$$

Мы видим, что коэффициенты при $g^{0 l}$ содержат комбинации

$$
\begin{aligned}
\frac{g^{0 m}}{g^{00}} \partial_{l} f_{m}^{A}+\partial_{l} f_{0}^{A} & =\frac{1}{g^{00}} \partial_{l} f^{0 A}-\frac{1}{g^{00}}\left(\partial_{l} g^{0 m} f_{m}^{A}+\partial_{l} g^{00} f_{0}^{A}\right), \\
\frac{g^{0 m}}{g^{00}} \partial_{0} f_{m}^{A}+\partial_{0} f_{0}^{A} & =\frac{1}{g^{00}} \partial_{0} f^{0 A}-\frac{1}{g^{00}}\left(\partial_{0} g^{0 m} f_{m}^{A}+\partial_{0} g^{00} f_{0}^{A}\right),
\end{aligned}
$$

и вторые слагаемые в правых частях обращаются в ноль при действии на них проектора $\Pi^{A B}$. В результате мы приходим к удовлетворительному выражению

$$
\Sigma=2 \sqrt{g} \Pi^{A B} \gamma^{k l}\left(\partial_{l} f_{k}^{A} \partial_{0} f^{0 B}-\partial_{l} f^{0 A} \partial_{0} f_{k}^{B}\right) .
$$

Перейдем теперь к 0-форме $\mathcal{H}$. Разделим сумму по $\rho$ и $\sigma$ на четыре в соответствии с разделением

$$
(\sigma, \rho)=(0,0),(m, 0),(0, n),(m, n) .
$$

Слагаемое $(0,0)$ исчезает вследствие антисимметрии. Слагаемые $(m, 0)$ и $(0, n)$ после замены значков суммирования совпадают и дают

$$
Q_{1}=2 \sqrt{g} \Pi^{A B} \gamma^{l n} g^{k 0}\left(\partial_{k} f_{0}^{A} \partial_{l} f_{n}^{B}-\partial_{l} f_{0}^{A} \partial_{k} f_{n}^{B}\right) .
$$

Наконец, слагаемое $(m, n)$ можно записать в виде

$$
Q_{2}=\sqrt{g} \Pi^{A B} g^{k m} g^{l n} S_{k l, m n}^{A B},
$$

где

$$
S_{k l, m n}^{A B}=\partial_{k} f_{m}^{A} \partial_{l} f_{n}^{B}-\partial_{k} f_{n}^{A} \partial_{l} f_{m}^{B}
$$


Выражая $g^{k m}$ через $\gamma^{k m}, g^{k 0}, g^{0 m}$, мы получаем $Q_{2}=Q_{3}+Q_{4}$, где

$$
Q_{3}=\sqrt{g} \Pi^{A B} \gamma^{k m} \gamma^{l n} S_{k l, m n}^{A B}, \quad Q_{4}=2 \sqrt{g} \frac{g^{0 k}}{g^{00}} \gamma^{l n} g^{0 m} S_{k l, m n}^{A B} .
$$

Собирая вместе $Q_{1}$ и $Q_{4}$ и используя свойство проектора $\Pi^{A B}$, уже объясненное выше, мы имеем

$$
Q_{1}+Q_{4}=2 \sqrt{g} \frac{g^{0 k}}{g^{00}} \Pi^{A B} \gamma^{l n}\left(\partial_{k} f^{0 A} \partial_{l} f_{n}^{B}-\partial_{l} f^{0 A} \partial_{k} f_{n}^{B}\right) .
$$

Итак, мы получили

$$
\mathcal{H}=T_{0}+T_{1}, \quad T_{1}=Q_{1}+Q_{4}, \quad T_{0}=Q_{3} .
$$

В формулы, как и в $\Sigma$, входят проектор $\Pi^{A B}$ и переменные $f_{k}^{A}, f^{0 A}, g^{i 0}, g^{0 n}$ и $g^{00}$.

Теперь мы должны преобразовать проектор. Имеем

$$
\begin{aligned}
\Pi^{A B} & =\delta^{A B}-g^{\mu \nu} f_{\mu}^{A} f_{\nu}^{B}= \\
& =\delta^{A B}-g^{i k} f_{i}^{A} f_{k}^{B}-g^{i 0} f_{i}^{A} f_{0}^{B}-g^{0 k} f_{0}^{A} f_{k}^{B}-g^{00} f_{0}^{A} f_{0}^{B}= \\
& =\delta^{A B}-\gamma^{i k} f_{i}^{A} f_{k}^{B}-\left(\frac{g^{0 i} g^{0 k}}{g^{00}} f_{i}^{A} f_{k}^{B}+g^{0 k} f_{0}^{A} f_{k}^{B}+g^{i 0} f_{i}^{A} f_{0}^{B}+f_{0}^{A} f_{0}^{B} g^{00}\right) .
\end{aligned}
$$

Два первых слагаемых в правой части определяют семимерный вертикальный проектор. Остальные слагаемые можно переписать в виде

$$
\frac{1}{g^{00}}\left(g^{0 i} f_{i}^{A}+g^{00} f_{0}^{A}\right)\left(g^{0 k} f_{k}^{B}+g^{00} f_{0}^{B}\right)=\frac{1}{g^{00}} f^{0 A} f^{0 B},
$$

так что окончательно получаем

$$
\Pi^{A B}=\delta^{A B}-\gamma^{i k} f_{i}^{A} f_{k}^{B}-\frac{1}{g^{00}} f^{0 A} f^{0 B} .
$$

Последнее слагаемое здесь дает одномерный проектор в силу условия нормировки для $f^{0 A}$.

Итак, мы выразили $\Sigma$ и $\mathcal{H}$ через выбранный набор динамических переменных. Подставим теперь последнее выражение для проектора $\Pi^{A B}$ в $\Sigma$ и $\mathcal{H}$. Начнем с $\Sigma$ и рассмотрим по очереди три выражения, соответствующие трем слагаемым в проекторе $\Pi^{A B}$. Первое имеет вид

$$
\Sigma_{1}=2 \sqrt{g} \gamma^{k l}\left(\partial_{l} f_{k}^{A} \partial_{0} f^{0 A}-\partial_{l} f^{0 A} \partial_{0} f_{k}^{A}\right) .
$$

Запишем множитель в круглых скобках как

$$
\partial_{0}\left(\partial_{l} f_{k}^{A} f^{0 A}\right)-\partial_{l}\left(\partial_{0} f_{k}^{A} f^{0 A}\right)=\partial_{0} \Omega_{k l}^{0}-\partial_{l} \Omega_{k 0}^{0} .
$$

Получаем

$$
\Sigma_{1}=2 \sqrt{g} \gamma^{k l}\left(\partial_{0} \Omega_{k l}^{0}-\partial_{l} \Omega_{k 0}^{0}\right) .
$$

Первое слагаемое здесь имеет форму, почти совпадающую с формой Дарбу. 
Перейдем ко второму вкладу в $\Sigma$ :

$$
\begin{aligned}
\Sigma_{2} & =-2 \sqrt{g} \gamma^{k l} \gamma^{m n}\left[\left(f_{m}^{A} \partial_{l} f_{k}^{A}\right)\left(f_{n}^{B} \partial_{0} f^{0 B}\right)-\left(f_{m}^{A} \partial_{l} f^{0 A}\right)\left(f_{n}^{B} \partial_{0} f_{k}^{B}\right)\right]= \\
& =2 \sqrt{g} \gamma^{k l} \gamma^{m n}\left(\omega_{m, k l} \Omega_{n 0}^{0}-\left(f_{n}^{B} \partial_{0} f_{k}^{B}\right) \Omega_{m l}^{0}\right) .
\end{aligned}
$$

Здесь я использовал ортогональность $f_{k}^{A}$ и $f_{0}^{A}$,

$$
f_{n}^{B} \partial_{0} f^{0 B}=-\partial_{0} f_{n}^{B} f^{0 B}=-\Omega_{n 0}^{0}, \quad f_{m}^{A} \partial_{l} f^{0 A}=-\partial_{l} f_{m}^{A} f^{0 A}=-\Omega_{m l}^{0},
$$

и ввел трехмерную связность $\omega_{m, k l}=f_{m}^{A} \partial_{l} f_{k}^{A}$.

Наконец, последний вклад в $\Sigma$ имеет вид

$$
\Sigma_{3}=-\sqrt{g} \gamma^{k l} \frac{1}{g^{00}}\left(\Omega_{k l}^{0} \partial_{0} g^{00}-\partial_{l} g^{00} \Omega_{k 0}^{0}\right) .
$$

Я использовал здесь соотношения

$$
f^{0 A} \partial_{0} f^{0 A}=\frac{1}{2} \partial_{0} g^{00}, \quad f^{0 A} \partial_{l} f^{0 A}=\frac{1}{2} \partial_{l} g^{00} .
$$

Соберем слагаемые, содержащие $\Omega_{k 0}^{0}$. Пусть

$$
\Lambda=2 \sqrt{g} \gamma^{k l}\left(-\partial_{l} \Omega_{k 0}^{0}+\omega_{k l}^{m} \Omega_{m 0}^{0}+\frac{1}{2} \frac{\partial_{l} g^{00}}{g^{00}} \Omega_{k 0}^{0}\right)
$$

сравним это выражение с

$$
\begin{aligned}
\partial_{l}\left(\sqrt{g} \gamma^{k l} \Omega_{k 0}^{0}\right) & =\partial_{l}\left(\frac{\sqrt{\gamma}}{\sqrt{g^{00}}} \gamma^{k l} \Omega_{k 0}^{0}\right)= \\
& =\sqrt{g}\left(\omega_{m l}^{m}-\frac{1}{2} \frac{\partial_{l} g^{00}}{g^{00}}\right) \gamma^{k l} \Omega_{k 0}^{0}+\sqrt{g} \partial_{l} \gamma^{k l} \Omega_{k 0}^{0}+\sqrt{g} \gamma^{k l} \partial_{l} \Omega_{k 0}^{0}
\end{aligned}
$$

Связность $\omega_{m l}^{k}$ совместна с метрикой $\gamma_{i k}$, так что

$$
\partial_{l} \gamma^{k l}+\omega_{m l}^{k} \gamma^{m l}+\omega_{m l}^{l} \gamma^{m k}=0
$$

В результате мы получаем

$$
\Lambda=-2 \partial_{l}\left(\sqrt{g} \gamma^{k l} \Omega_{k 0}^{0}\right)+2 \sqrt{g} \gamma^{k l} \Omega_{k 0}^{0}\left(\omega_{m l}^{m}-\omega_{l m}^{m}\right) .
$$

Последнее слагаемое исчезает в силу упомянутых выше уравнений движения. Действительно, мы имеем

$$
\begin{aligned}
\Omega_{i k}^{m} & =f^{m A} \partial_{k} f_{i}^{A}=g^{m \sigma} f_{\sigma}^{A} \partial_{k} f_{i}^{A}= \\
& =\left(\gamma^{m n}+\frac{g^{m 0} g^{n 0}}{g^{00}}\right) f_{n}^{A} \partial_{k} f_{i}^{A}+g^{m 0} f_{0}^{A} f_{i}^{A}=\omega_{i k}^{m}+\frac{g^{m 0}}{g^{00}} \Omega_{i k}^{0},
\end{aligned}
$$

и $\omega_{i k}^{m}$ симметрично по отношению к перестановке $i \leftrightarrow k$ вместе с $\Omega_{i k}^{m}$ и $\Omega_{i k}^{0}$.

Итак, мы получили, что $\Lambda$ представляет собой выражение типа дивергенции и может быть опущено в действии. 
Теперь рассмотрим первое слагаемое в $\Sigma_{2}$. В силу симметрии $\Omega_{k l}^{0}$ мы можем записать

$$
\gamma^{k l} \gamma^{m n} \Omega_{m l}^{0}\left(f_{n}^{A} \partial_{0} f_{k}^{A}\right)=\frac{1}{2} \gamma^{k l} \gamma^{m n}\left(f_{n}^{A} \partial_{0} f_{k}^{A}+f_{k}^{A} \partial_{0} f_{n}^{A}\right) \Omega_{m l}^{0}=-\frac{1}{2} \partial_{0} \gamma^{m l} \Omega_{m l}^{0} .
$$

В результате всех этих преобразований мы получаем

$$
\Sigma=\sqrt{g}\left(2 \gamma^{k l} \partial_{0} \Omega_{k l}+\partial_{0} \gamma^{k l} \Omega_{k l}^{0}-\gamma^{k l} \Omega_{k l} \frac{\partial_{0} g^{00}}{g^{00}}\right)=q^{k l} \partial_{0} \Pi_{k l}+\partial_{0}\left(\sqrt{g} \gamma^{k l} \Omega_{k l}\right),
$$

где

$$
q^{k l}=\gamma \gamma^{k l}, \quad \Pi_{k l}=\frac{1}{\sqrt{\gamma g^{00}}} \Omega_{k l}^{0} .
$$

Полную производную по $x^{0}$ можно исключить из действия. Таким образом, 1-форма $\Sigma$ получает явно канонический вид:

$$
\Sigma=q^{i k} d \Pi_{i k}
$$

с каноническими переменными $q^{i k}$ и $\Pi_{i k}$. Их конформные веса, равные +1 для $q^{i k}$ и $-1 / 2$ для $\Pi_{i k}$, естественно связаны с нормировкой $\delta$-функции. Эти канонические пары впервые появились в работе [13]. В работах [2] и [14] использовались другие веса.

Теперь перейдем к 0-форме $\mathcal{H}$. Начнем с выражения для $T_{1}$,

$$
T_{1}=2 \sqrt{g} \frac{g^{k 0}}{g^{00}} \Pi^{A B} \gamma^{l m}\left(\partial_{k} f^{0 A} \partial_{l} f_{m}^{B}-\partial_{l} f^{0 A} \partial_{k} f_{m}^{B}\right),
$$

и опять рассмотрим три вклада в соответствии с представлением проектора $\Pi^{A B}$. В первом слагаемом используем равенства

$$
\partial_{k} f^{0 A} \partial_{l} f_{m}^{A}-\partial_{l} f^{0 A} \partial_{k} f_{m}^{A}=\partial_{k}\left(f^{0 A} \partial_{l} f_{m}^{A}\right)-\partial_{l}\left(f^{0 A} \partial_{k} f_{m}^{A}\right)=\partial_{k} \Omega_{m l}^{0}-\partial_{l} \Omega_{m k}^{0},
$$

во втором получим

$$
-\gamma^{p q}\left[\left(f_{p}^{A} \partial_{k} f^{0 A}\right)\left(f_{q}^{B} \partial_{l} f_{m}^{B}\right)-\left(f_{p}^{A} \partial_{l} f^{0 A}\right)\left(f_{q}^{B} \partial_{k} f_{m}^{B}\right)\right]=\omega_{m l}^{p} \Omega_{p k}^{0}-\omega_{m k}^{p} \Omega_{p l}^{0},
$$

а третье дает

$$
-\frac{1}{g^{00}}\left[\left(f^{0 A} \partial_{k} f^{0 A}\right)\left(f^{0 B} \partial_{l} f_{m}^{B}\right)-\left(f^{0 A} \partial_{l} f^{0 A}\right)\left(f^{0 B} \partial_{k} f_{m}^{B}\right)\right]=-\frac{1}{2} \frac{\partial_{k} g^{00}}{g^{00}} \Omega_{m l}^{0}+\frac{1}{2} \frac{\partial_{l} g^{00}}{g^{00}} \Omega_{m k}^{0} .
$$

Собрав все слагаемые вместе, имеем

$$
T_{1}=2 \frac{g^{k 0}}{g^{00}} \sqrt{g} \gamma^{l m}\left[\partial_{k} \Omega_{m l}^{0}-\partial_{l} \Omega_{m k}^{0}-\frac{1}{2} \frac{\partial_{k} g^{00}}{g^{00}} \Omega_{m l}^{0}+\frac{1}{2} \frac{\partial_{l} g^{00}}{g^{00}} \Omega_{m k}^{0}+\omega_{m l}^{p} \Omega_{p k}^{0}+\omega_{m n}^{p} \Omega_{p l}^{0}\right] .
$$

Используя симметрию $\omega_{m l}^{p}$ и $\Omega_{p l}^{0}$, запишем $T_{1}$ в виде $T_{1}=\lambda^{k} \mathcal{H}_{k}$, где введены множители Лагранжа $\lambda^{k}=g^{0 k} / g^{00}$ и связи

$$
\mathcal{H}_{k}=2\left[\nabla_{k}\left(q^{m l} \Pi_{m l}\right)-\nabla_{l}\left(q^{m l} \Pi_{m k}\right)\right] .
$$


Здесь использованы трехмерные ковариантные производные

$$
\nabla_{k} T_{m}^{l}=\partial_{k} T_{m}^{l}+\omega_{m k}^{l} T_{m}^{n}-\omega_{m k}^{n} T_{n}^{l}
$$

Теперь перейдем к $T_{0}$. Трехмерный проектор ${ }^{3} \Pi^{A B}$, участвующий в $\Pi^{A B}$, дает вклад

$$
\sqrt{g} \gamma^{k m} \gamma^{\ln 3} \Pi^{A B} S_{k l, m n}^{A B}=\sqrt{g} S^{(3)},
$$

где $S^{(3)}$ - скалярная кривизна метрики $\gamma_{i k}$ и связности $\omega_{n l}^{m}$. Последнее слагаемое в $\Pi^{A B}$ дает

$$
-\frac{f^{0 A} f^{0 B}}{g^{00}} S_{k l, m n}^{A B}=-\frac{1}{g^{00}}\left(\Omega_{m k}^{0} \Omega_{n l}^{0}-\Omega_{n k}^{0} \Omega_{m l}^{0}\right) .
$$

Собирая все слагаемые вместе, получаем

$$
T_{0}=\lambda^{0} \mathcal{H}_{0},
$$

где множитель Лагранжа $\lambda^{0}$ дается выражением

$$
\lambda^{0}=\frac{\sqrt{g}}{\gamma}=\frac{1}{\sqrt{g^{00}} \gamma}=\frac{1}{\sqrt{g} g^{00}},
$$

а связь $\mathcal{H}_{0}$ выглядит следующим образом:

$$
\mathcal{H}_{0}=\gamma S^{(3)}-q^{k m} q^{l n}\left(\Pi_{k m} \Pi_{l n}-\Pi_{l m} \Pi_{k n}\right) .
$$

Итак, мы выразили действие через динамические переменные $q^{i k}, \Pi_{i k}$ и множители Лагранжа $\lambda^{0}, \lambda^{k}$. Другими словами, из 40 динамических компонент $f_{\alpha}^{A}$ у нас осталось 16, а именно 6 канонических пар и четыре множителя Лагранжа, и действие имеет вид

$$
A=\int d^{3} x \int\left(q^{i k} \Pi_{i k}+\lambda^{0} \mathcal{H}_{0}+\lambda^{k} \mathcal{H}_{k}\right) d t
$$

эквивалентный формулам из работ [2] и [14].

\section{5. О(6)-СИММЕТРИЯ}

Здесь я приведу предварительное обсуждение возможного обобщения теории Эйнштейна путем введения новых полей. Естественная идея состоит в использовании вертикального пространства, дополняющего в $\mathbb{R}^{10}$ базис, образованный полями $f_{\mu}^{A}$. Пусть $e_{a}^{A}, a=1, \ldots, 6,-$ ортонормированная система векторов, ортогональных векторам $f_{\mu}^{A}$ :

$$
f_{\mu}^{A} e_{a}^{A}=0, \quad e_{a}^{A} e_{b}^{A}=\delta_{a b} .
$$

Будем считать набор $e_{a}^{A}$ скалярами по отношению к координатным преобразованиям $\delta e_{a}^{A}=-\epsilon^{\lambda} \partial_{\lambda} e_{a}^{A}$. Тогда соотношения ортогональности ковариантны. Они также инвариантны по отношению к локальным вращениям $e_{a}^{A} \rightarrow O_{a b} e_{b}^{A}, f_{\mu}^{A} \rightarrow f_{\mu}^{A}$, где $O_{a b}$ - ортогональные матрицы, образующие группу $O(6)$.

Проектор $\Pi^{A B}$ записывается как $\Pi^{A B}=e_{a}^{A} e_{a}^{B}$, что позволяет переписать основной лагранжиан $\mathcal{L}$ в виде

$$
\mathcal{L}=\sqrt{g}\left(f^{\mu A} f^{\nu B}-f^{\nu A} f^{\mu B}\right) \partial_{\mu} e_{a}^{A} \partial_{\nu} e_{a}^{B},
$$


напоминающем сигма-модель. Производные $\partial_{\mu} e_{a}^{A}$ здесь можно заменить на $O(6)$-ковариантные, если ввести связность $\Gamma_{\mu, a b}=\partial_{\mu} e_{a}^{A} e_{b}^{A}$. Действительно,

$$
\nabla_{\mu}^{\Gamma} e_{a}^{A}=\partial_{\mu} e_{a}^{A}-\Gamma_{\mu, a b} e_{b}^{A}=\partial_{\mu} e_{a}^{B}\left(\delta^{A B}-e_{b}^{A} e_{b}^{B}\right)=\partial_{\mu} e_{a}^{B} f^{\sigma B} f_{\sigma}^{A}
$$

и, таким образом,

$$
f^{\nu A} \nabla_{\mu}^{\Gamma} e_{a}^{A}=f^{\nu A} \partial_{\mu} e_{a}^{A}
$$

Следующий естественный шаг - введение набора скалярных полей $X^{A}$ таких, что $\delta X^{A}=-\epsilon^{\lambda} \partial_{\lambda} X^{A}$, и соответствующих горизонтальных и вертикальных компонент векторного поля $Y_{\mu}=X^{A} f_{\mu}^{A}$ и набора скаляров $Z_{a}=e_{a}^{A} X^{a}$. Найдем их ковариантные производные:

$$
\begin{aligned}
\nabla_{\mu} Y_{\nu} & =\partial_{\mu}\left(f_{\nu}^{A} X^{A}\right)-f^{\beta B} \partial_{\mu} f_{\nu}^{B} f_{\beta}^{A} X^{A}=f_{\nu}^{A} \partial_{\mu} X^{A}+\partial_{\mu} f_{\nu}^{B}\left(\delta^{A B}-f^{\beta B} f_{\rho}^{A}\right) X^{A}= \\
& =f_{\nu}^{A} \partial_{\mu} X^{A}+\Pi^{A B} \partial_{\mu} f_{\nu}^{B} X^{A}=f_{\nu}^{A} \partial_{\mu} X^{A}+b_{\nu \mu}^{A} X^{A} .
\end{aligned}
$$

Далее,

$$
\begin{aligned}
\nabla_{\mu}^{\Gamma} Z_{a} & =\partial_{\mu} Z_{a}-\Gamma_{\mu, a b} Z_{b}=e_{a}^{A} \partial_{\mu} X^{A}+\partial_{\mu} e_{a}^{A}\left(\delta^{A B}-\Pi^{A B}\right) X^{B}= \\
& =e_{a}^{A}\left(\partial_{\mu} X^{A}-b_{\mu}^{\sigma A} Y_{\sigma}\right) .
\end{aligned}
$$

Заметим, что в членах без производных от $X^{A}$ участвует вертикальная компонента $\Pi^{A B} X^{B}$ в $\nabla_{\mu} Y_{\nu}$ и горизонтальныя компонента $Y_{\nu}$ в $\nabla_{\mu}^{\Gamma} Z_{a}$. Оба этих слагаемых содержат тензор $b_{\nu \mu}^{A}$. Вклад в ковариантную комбинацию

$$
\sqrt{g}\left(g^{\mu \alpha} g^{\nu \beta}-g^{\mu \beta} g^{\nu \alpha}\right) \nabla_{\mu} Y_{\alpha} \nabla_{\nu} Y_{\beta}
$$

не содержащий производных от $X^{A}$, имеет вид

$$
\sqrt{g}\left(b_{\mu}^{\mu A} b_{\nu}^{\nu B}-b_{\mu}^{\nu A} b_{\nu}^{\mu B}\right) X^{A} X^{B},
$$

и мы получим гравитационное действие, если предположим, что у нас есть аномальная функция Грина

$$
\left\langle X^{A} X^{B}\right\rangle=\frac{1}{\kappa^{2}} \Pi^{A B} .
$$

Конечно, в настоящее время это замечание есть всего лишь спекуляция. Дальнейшая работа должна показать, насколько эта идея или ее модификация имеют смысл. На этом замечании о такой перспективе я закончу данную работу.

Благодарности. Я хочу поблагодарить В. Франке и в особенности С. Пастона за критическое обсуждение этой работы и С. Дезера за моральную поддержку. Работа выполнена при частичной поддержке РФФИ (гранты № 08-01-00638, 09-01-93108-НЦНИЛ) и Программы президиума РАН "Математические проблемы нелинейной динамики".

\section{Список литературы}

[1] A. Einstein, Ann. Phys., 354:7 (1916), 769-822; 14:Suppl. 1 (2005), 517-571.

[2] P. A. M. Dirac, Proc. Roy. Soc. A, 246:1246 (1958), 333-343.

[3] Л. Д. Фаддеев, В. Н. Попов, УФН, 111 (1973), 427-450. 
[4] S. Weinberg, Phys. Rev. Lett., 19:21 (1967), 1264-1266.

[5] A. Salam, "Weak and Electromagnetic Interactions", Elementary Particle Theory, Proc. Nobel Symposium (Lerum, Sweden, 1968), ed. N. Svartholm, Almquist and Wiksell, Stockholm, 1968, 367-377.

[6] S. L. Adler, Rev. Modern Phys., 54:3 (1982), 729-766.

[7] S. Deser, F. A. E. Pirani, D. C. Robinson, Phys. Rev. D, 14:12 (1976), 3301-3303.

[8] С. А. Пастон, В. А. Франке, ТМФ, 153:2 (2007), 271-288, arXiv: 0711.0576.

[9] L. D. Faddeev, New variables for the Einstein theory of gravitation, arXiv: 0911.0282.

[10] L. D. Faddeev, $3+1$ decomposition in the new action for the Einstein theory of gravitation, arXiv: 1003.2311.

[11] L. D. Faddeev, R. Jackiw, Phys. Rev. Lett., 60:17 (1988), 1692-1694.

[12] Л. Д. Фаддеев, УФН, 136:3 (1982), 435-457.

[13] J. S. Schwinger, Phys. Rev., 130:3 (1963), 1253-1258.

[14] R. L. Arnowitt, S. Deser, C. W. Misner, Phys. Rev., 117:6 (1960), 1595-1602.

Поступила в редакцию 6.09.2010 